\title{
Immunephenotype Predicts Response to Vedolizumab: Integrating Clinical and Biochemical Biomarkers in the Treatment of Inflammatory Bowel Diseases
}

\author{
Stefano Fiorucci ${ }^{1} \cdot$ Michele Biagioli $^{1}$ - Eleonora Distrutti ${ }^{2}$
}

Published online: 2 April 2018

(c) Springer Science+Business Media, LLC, part of Springer Nature 2018

In the past decades, biological agents (engineered monoclonal antibodies [mAbs]) have emerged as essential therapy for moderate-to-severe inflammatory bowel disease (IBD). More recently, the $\mathrm{mAb}$ tumor necrosis factor- $\alpha$ antagonists (anti-TNF $\alpha$ ), infliximab, adalimumab, golimumab, and certolizumab pegol have been supplemented by newer clinically approved biological agents that target different inflammatory pathways. Vedolizumab is a humanized $\mathrm{mAb}$ and the first in class of selective anti- $\alpha 4 \beta 7$ integrin that has gained approval for the treatment of moderate-to-severe IBD in adult patients who fail to respond to, lose response to, or are intolerant to conventional therapies (corticosteroids and immunomodulators) or to TNF $\alpha$ mAbs [1]. Natalizumab is another anti- $\alpha 4$ $\mathrm{mAb}$ that in contrast to vedolizumab binds both the $\alpha 4 \beta 7$ and- $\alpha 4 \beta 1$ heterodimers and is therefore less selective. The $\alpha 4 \beta 7$ integrin an adhesion molecule expressed on a variety of circulating leukocytes, including cluster of differentiation (CD) $4^{+}$and $\mathrm{CD} 8^{+}$naive $\mathrm{T}$ cells, $\mathrm{CD} 4^{+}$and $\mathrm{CD} 8^{+}$memory $T$ cells, $B$ cells, eosinophils, natural killer (NK) cells, that is essential for the migration these cells from the systemic circulation to the inflamed intestine. The counterpart of $\alpha 4 \beta 7$ integrin is primarily, but not exclusively, represented by the mucosal addressin cell adhesion molecule-1 (MAdCAM-1), whose expression is abundant in intestinal endothelial cells (Fig. 1a, b). Because the $\alpha 4 \beta 7-M A d C A M-1$ interaction is highly restricted to the intestine, vedolizumab appears to selectively target recruitment of inflammatory cells to the gastrointestinal tract. The success of vedolizumab in treating IBD has facilitated the development of additional treatments

Stefano Fiorucci

Stefano.Fiorucci@unipg.it

1 Section of Gastroenterology, Department of Surgical and Biomedical Science, University of Perugia, Piazza Lucio Severi 1, S. Andrea delle Fratte, 06132 Perugia, Italy

2 Azienda Ospedaliera di Perugia, Perugia, Italy that target the $\beta 7$ integrin (etrolizumab) or MAdCAM-1 (PF-00547659).

In preclinical models, vedolizumab effectively reduced leukocyte trafficking toward the intestine, thus reducing inflammation and immune dysfunction. These findings were confirmed by results of Phase III trials, in which the anti- $\alpha 4 \beta 7$ antagonist successfully induced and maintained remission in patients with Crohn's disease (CD) and ulcerative colitis (UC) [2-4]. Nevertheless, similar to anti-TNF $\alpha$ agents, a significant proportion of patients $(\approx 40 \%)$ failed to reach or to maintain remission in response to vedolizumab. In real-word cohorts, the primary response rate to vedolizumab is effectively similar in UC and CD patients, $40-60 \%$ of patients $[5,6]$. Lack of response or need for dose optimization occurs in 40-60\% of IBD patients during the maintenance phase $[7,8]$. Due to the above factors, the place of vedolizumab in the management of IBD remains unclear, particularly taking into account the utility of anti-integrin therapy in the context of currently available biological therapies. In patients with less severe disease, as assessed by clinical and biochemical markers and naïve to anti-TNF $\alpha$ therapy, those with higher circulating concentrations of vedolizumab at induction are more likely to respond to treatment. The severity of disease at baseline is a robust predictor of response to vedolizumab in both UC and CD patients. Thus, while a higher response rate was observed in patients with a baseline Mayo score $<9$ and Crohn's disease activity index (CDAI) score $\leq 330$ at 6 and 54 weeks [2, 3], others have reported a less favorable outcome in patients with Harvey-Bradshaw index (HBI) score $>10$ and/or a Mayo score $>9$ at 54 weeks [7]. Additionally, a history of smoking and active perianal disease were inverse predictors for clinical remission during maintenance therapy [8]. Further on, patients with severe systemic and bowel inflammation, as measured by higher concentrations of the nonspecific inflammatory biomarkers C-reactive protein (C-RP), leukocytosis, and fecal calprotectin are less likely to reach a 

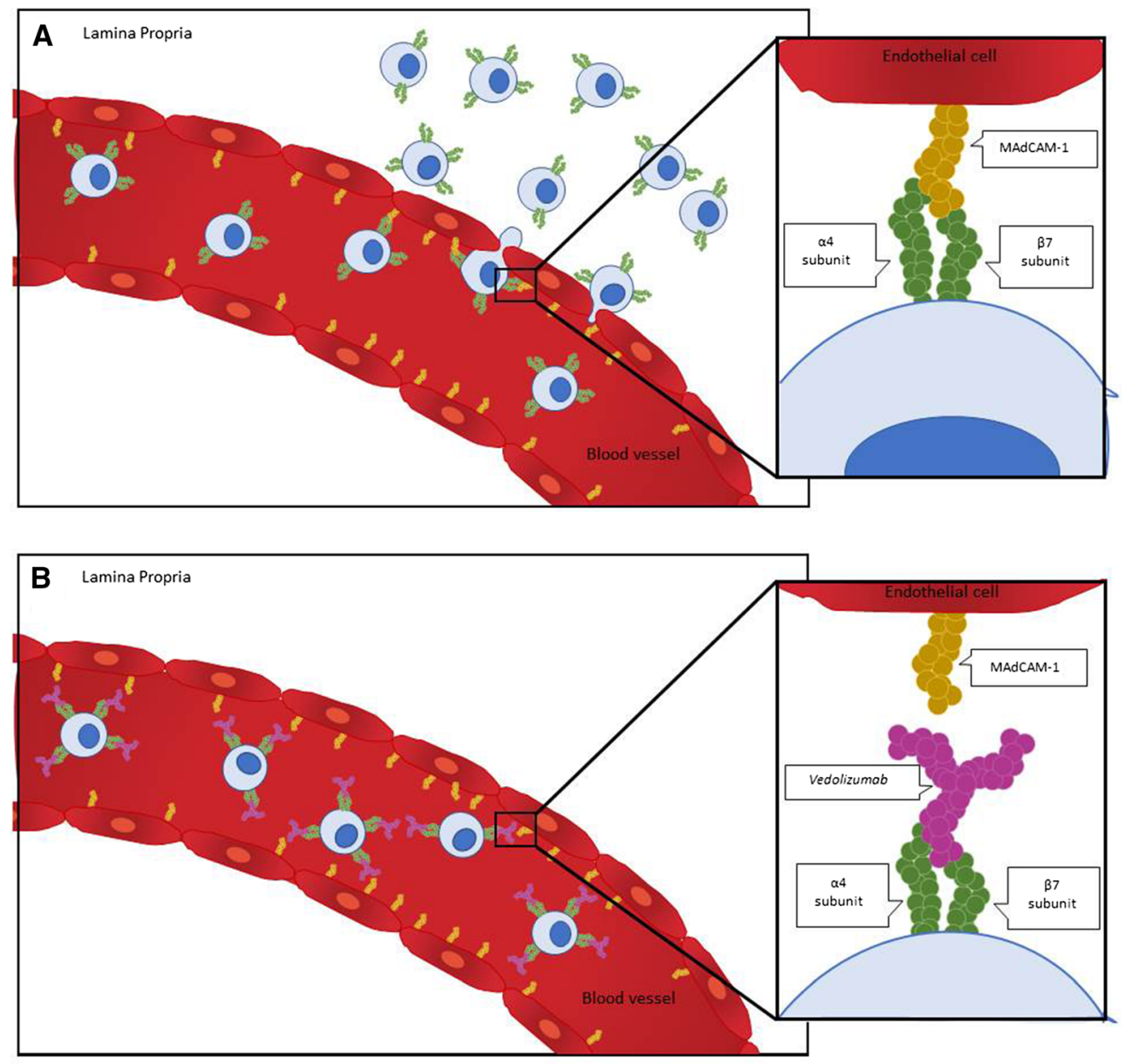

C Cells with high levels of $\alpha 4 \beta 7$ expression in responders to vedilizumab

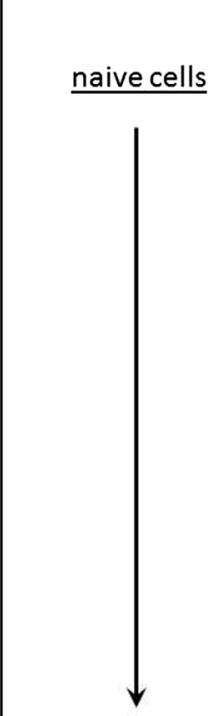

highly differentiated cells
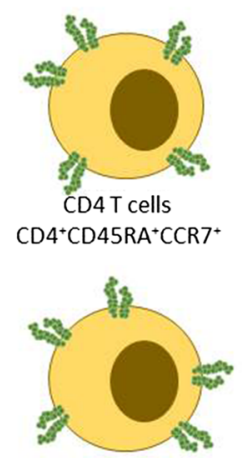

CD4 $\mathrm{T}_{\mathrm{EM}}$ cells $\mathrm{CD}^{+} \mathrm{CD} 45 \mathrm{RA} \cdot \mathrm{CCR} 7^{+}$

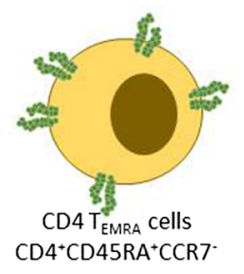

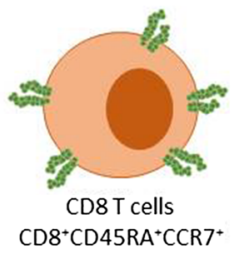
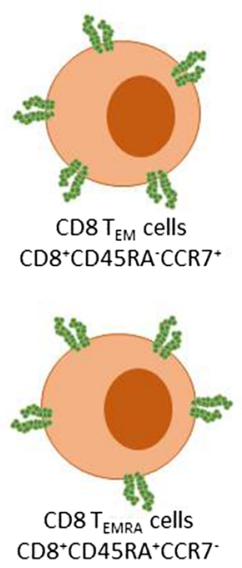

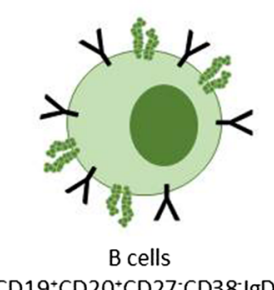

$\mathrm{CD} 19^{+} \mathrm{CD} 20^{+} \mathrm{CD} 27^{-} \mathrm{CD} 38^{-} \cdot \mathrm{Ig}^{+}$ 
4Fig. 1 Role of $\alpha 4 \beta 7$ integrin in the regulation of leukocyte trafficking in the intestine and identification of markers predictive of outcome. a Interaction between $\alpha 4 \beta 7$ and MAdCAM- 1 at the endothelial interface in the intestine. b Mechanism of action of vedolizumab. c Immunephenotype of IBD patients responders to vedolizumab (from ref. [13]) . High expression of $\alpha 4 \beta 7$ in peripheral blood mononuclear cell subsets (memory $T$ cells, $T_{\mathrm{EMRA}}$, and NK cells) at baseline predicts response to vedolizumab

clinical response or to maintain remission when treated with vedolizumab [9, 10]. Elevated C-RP at initiation is also associated with a higher probability of vedolizumab discontinuation due to lack or loss of response.

Additional outcome indicators in CD patients appear to be gender, age, and disease duration, with male, younger patients (age $<35$ years), and patients with shorter disease duration ( $<7$ years) having superior response rates. Other factors that predict a favorable outcome are no use of anti-TNF prior to starting vedolizumab [2, 3] since the probability of a favorable clinical response is significantly greater in patients naive of biological agents and with early response to treatment [7]. In contrast, predictors of lower rates of response to vedolizumab are the need for steroid co-medication to achieve remission or the need for immunosuppressants to maintain remission [11]. Nonetheless, the question of whether to add or continue thiopurines in combination with vedolizumab remains unclear. Finally, there is evidence that pharmacokinetic factors contribute to the clinical response. Higher vedolizumab concentrations at induction are associated with higher clinical remission rates [12]. It has been suggested that low serum albumin concentrations and high body weight might have a negative impact on vedolizumab pharmacokinetics [11].

While all the above data emphasize general factors that might predict the outcome of a favorable clinical response to vedolizumab, it is unclear whether the immunophenotype of IBD patients prior or during the treatment with anti- $\alpha 4 \beta 7$ can predict the outcome. In this issue of Digestive Diseases and Sciences, Dr. Boken et al. [13] report on one of the first attempt to study $\alpha 4 \beta 7$ expressed on peripheral blood mononuclear cells (PMBC) as a predictor of clinical outcome in IBD patients receiving vedolizumab. The study included 26 subjects (15 with CD, 11 with UC or indeterminate colitis). All subjects but three were anti-TNF experienced. Response, defined by endoscopic (HBI score) or clinical criteria, was achieved in 14 subjects. Nonresponse (12 subjects) was determined by failure to wean steroids in six and a lack of endoscopic improvement in the other six subjects. All non-responders had follow-up evaluations between 90 and 180 days after vedolizumab induction that failed to meet criteria for response.

Blood samples were collected before and through the study, and expression of $\alpha 4 \beta 7$ on several PMBC subsets was measured by flow cytometry. The authors identified several candidate biomarkers that were linked to clinical outcome (Fig. 1c). In general, pretreatment $\alpha 4 \beta 7$ expression on multiple $T, B$, and NK subsets was higher in responders than non-responders to vedolizumab. More specifically, a significantly higher per cell expression of $\alpha 4 \beta 7$ on naïve CD4 and CD8 $T$ cells (CD45RA+CCR7+) and B cells (CD19+CD20+CD27-CD38-IgD+) was predictive of a superior response. Patients who responded to vedolizumab also had a higher percentage of $\alpha 4 \beta 7$-expressing CD 4 and CD8 effector memory $T\left(T_{\mathrm{MEM}}\right)$ cells, and a subset of highly differentiated effector CD4 and CD8 T cells, called the terminal effector memory ( $\left.T_{\text {EMRA }}\right) T$ cells (CD45RA+CCR7-), showed an even more significant difference in percentage of $\alpha 4 \beta 7$ expression at baseline between responders and nonresponders (Fig. 1c). Furthermore, the percentage of NK cells (CD56+CD161+) expressing $\alpha 4 \beta 7$ prior to treatment with vedolizumab was significantly higher in responders compared to non-responders. The authors concluded that the amount of $\alpha 4 \beta 7$ expression on $T_{\text {EMRA }}$ and NK cells at baseline provided the best discrimination between responders and non-responders to vedolizumab. Moreover, both serum vedolizumab concentrations and $\alpha 4 \beta 7$ receptor saturation at trough were associated with response to therapy before and during vedolizumab therapy. Interestingly, serum vedolizumab concentrations declined more rapidly in nonresponders than in responders to vedolizumab, indicating that $\alpha 4 \beta 7$ receptors in non-responders were less saturated at vedolizumab concentrations that saturated the receptors in responders. Taken together, these findings suggest that non-responders have either a higher rate of $T$ cell turnover from the bone marrow or increased $\alpha 4 \beta 7$ turnover, perhaps via differences in integrin internalization and intracellular trafficking.

Should these results inform prescribing practices for vedolizumab in real-world patients? In other words, should the PMBC of patients for whom vedolizumab therapy is planned be tested for expression of $\alpha 4 \beta 7$ ? The answer at this stage is likely negative. Indeed, the study needs to be taken in an appropriate context: First, the cohort is small, with only 26 patients included and the two arms consist of only 14 and 12 subjects; second, since almost all subjects were anti-TNF experienced, the authors' findings might not generalize to TNF-naïve patients; third, no attempt was made to correlate $\alpha 4 \beta 7$ expression on leukocyte subsets with validated clinical response biomarkers such as C-RP, leukocytosis, or fecal calprotectin; and fourth, the blood samples were not collected at standard times, which in clinical trials is generally considered a measure of quality of data.

Even though the study needs further validation in larger cohorts, it provides novel insights that could be taken into account to further define the best personalized approach to target inflammation and immune dysfunction in IBD patients. 


\section{References}

1. Zundler S, Becker E, Weidinger C, Siegmund B. Anti-adhesion therapies in inflammatory bowel disease-molecular and clinical aspects. Front Immunol. 2017;28:891. https://doi.org/10.3389/ fimmu.2017.00891.

2. Feagan BG, Rutgeerts P, Sands BE, et al. Vedolizumab as induction and maintenance therapy for ulcerative colitis. $N$ Engl J Med. 2013;369:699-710.

3. Sandborn WJ, Feagan BG, Rutgeerts P, et al. Vedolizumab as induction and maintenance therapy for Crohn's disease. $N$ Engl J Med. 2013;369:711-721.

4. Sands BE, Feagan BG, Rutgeerts P, et al. Effects of vedolizumab induction therapy for patients with Crohn's disease in whom tumor necrosis factor antagonist treatment failed. Gastroenterology. 2014;147:618-627.

5. Shelton E, Allegretti JR, Stevens B, et al. Efficacy of vedolizumab as induction therapy in refractory IBD patients: a multicenter cohort. Inflamm Bowel Dis. 2015;21:2879-2885.

6. Baumgart DC, Bokemeyer B, Drabik A, Stallmach A, Schreiber $\mathrm{S}$, Vedolizumab Germany Consortium. Vedolizumab induction therapy for inflammatory bowel disease in clinical practice-a nationwide consecutive German cohort study. Aliment Pharmacol Ther. 2016;43:1090-1110.

7. Amiot A, Serrero M, Peyrin-Biroulet L, et al. One-year effectiveness and safety of vedolizumab therapy for inflammatory bowel disease: a prospective multicentre cohort study. Aliment Pharmacol Ther. 2017;46:310-321.

8. Dulai PS, Singh S, Jiang X, et al. The real-world effectiveness and safety of vedolizumab for moderate-severe Crohn's disease: results from the US VICTORY consortium. Am J Gastroenterol. 2016;111:1147-1155.

9. Amiot A, Filippi J, Roblin X, et al. Effectiveness and safety of vedolizumab induction therapy for patients with inflammatory bowel disease. Clin Gastroenterol Hepatol. 2016;14:1593-1601.

10. Stallmach A, Langbein C, Atreya R, et al. Vedolizumab provides clinical benefit over 1 year in patients with active inflammatory bowel disease - a prospective multicenter observational study. Aliment Pharmacol Ther. 2016;44:1199-1212.

11. Rosario M, Dirks NL, Gastonguay MR, et al. Population pharmacokinetics-pharmacodynamics of vedolizuma $b$ in patients with ulcerative colitis and Crohn's disease. Aliment Pharmacol Ther. 2015;42:188-202.

12. Rosario M, French JL, Dirks NL, et al. Exposure-efficacy relationships for vedolizumab induction therapy in patients with ulcerative colitis or Crohn's disease. J Crohn's Colitis. 2017;11:921-929.

13. Boden KE, Shows DM, Chiorean MV, Lord JD. Identification of candidate biomarkers associated with response to vedolizumab in inflammatory bowel disease. Dig Dis Sci. (Epub ahead of print). https://doi.org/10.1007/s10620-018-4924-8. 